\title{
Article \\ Green Synthesis of Nickel Oxide Nanoparticles from Berberis balochistanica Stem for Investigating Bioactivities
}

\author{
Siraj Uddin ${ }^{1,2}$, Luqman Bin Safdar ${ }^{3,4, *(D)}$, Saeed Anwar ${ }^{1}$, Javed Iqbal ${ }^{1,5}$, Sabiha Laila ${ }^{6}$, Banzeer Ahsan Abbasi ${ }^{1}$, \\ Muhammad Saqib Saif ${ }^{7}$, Musrat Ali ${ }^{1}$, Abdul Rehman ${ }^{8}{ }^{1}$, Abdul Basit ${ }^{9}$, Yong Wang ${ }^{9, *}$ \\ and Umar Masood Quraishi ${ }^{1, * \mathbb{D}}$
}

\section{check for} updates

Citation: Uddin, S.; Safdar, L.B.; Anwar, S.; Iqbal, J.; Laila, S.; Abbasi, B.A.; Saif, M.S.; Ali, M.; Rehman, A.; Basit, A.; et al. Green Synthesis of Nickel Oxide Nanoparticles from Berberis balochistanica Stem for Investigating Bioactivities. Molecules 2021, 26, 1548. https://doi.org/ $10.3390 /$ molecules 26061548

Academic Editors: Tetsu Yonezawa and Antonio Zuorro

Received: 14 January 2021

Accepted: 4 March 2021

Published: 11 March 2021

Publisher's Note: MDPI stays neutral with regard to jurisdictional claims in published maps and institutional affiliations.

Copyright: (c) 2021 by the authors. Licensee MDPI, Basel, Switzerland. This article is an open access article distributed under the terms and conditions of the Creative Commons Attribution (CC BY) license (https:/ / creativecommons.org/licenses/by/ $4.0 /)$.
1 Department of Plant Sciences, Faculty of Biological Sciences, Quaid-i-Azam University, Islamabad 45320, Pakistan; usiraj@bs.qau.edu.pk (S.U.); saeedanwar@bs.qau.edu.pk (S.A.); jiqbal@bs.qau.edu.pk (J.I.); banzeer.abbasi@bs.qau.edu.pk (B.A.A.); musrat.ali@bs.qau.edu.pk (M.A.)

2 Plant Breeding Institute, University of Sydney, Narellan, NSW 2567, Australia

3 School of Biosciences, Sutton Bonington Campus, University of Nottingham, Sutton Bonington, Leicestershire LE12 5RD, UK

4 School of Agriculture, Food and Wine, Waite Research Institute, University of Adelaide, Glen Osmond, SA 5064, Australia

5 Department of Botany, Bacha Khan University, Charsadda 24420, Pakistan

6 Department of Botany, Sardar Bahadur Khan Women's University, Quetta 1800, Pakistan; sabiha.qumber786@gmail.com

7 Department of Biochemistry, The Islamia University of Bahawalpur, Bahawalpur 63100, Pakistan; saqibsaif.qau@gmail.com

8 Centre of Excellence in Solid State Physics, Quaid I Azam Campus, University of Punjab, Lahore 54000, Pakistan; abdul.bsphy510@iiu.edu.pk

9 Department of Plant Pathology, Agriculture College, Guizhou University, Guiyang 550025, China; basit@gzu.edu.cn

* Correspondence: luqman.safdar@nottingham.ac.uk (L.B.S.); yongwangbis@aliyun.com (Y.W.); umasood@qau.edu.pk (U.M.Q.)

Abstract: Green synthesis of nanomaterials is advancing due to its ease of synthesis, inexpensiveness, nontoxicity and renewability. In the present study, an eco-friendly biogenic method was developed for the green synthesis of nickel oxide nanoparticles (NiONPs) using phytochemically rich Berberis balochistanica stem (BBS) extract. The BBS extract was rich in phenolics, flavonoids and berberine. These phytochemicals successfully reduced and stabilised the $\mathrm{NiNO}_{3}$ (green) into NiONPs (greenishgray). BBS-NiONPs were confirmed by using UV-visible spectroscopy (peak at $305 \mathrm{~nm}$ ), X-ray diffraction (size of $31.44 \mathrm{~nm}$ ), Fourier transform infrared spectroscopy (identified -OH group and Ni-O formation), energy dispersive spectroscopy (showed specified elemental nature) and scanning electron microscopy (showed rhombohedral agglomerated shape). BBS-NiONPs were exposed to multiple in vitro bioactivities to ascertain their beneficial biological applications. They exhibited strong antioxidant activities: total antioxidant capacity (64.77\%) and 2, 2-diphenyl-1-picrylhydrazyl (71.48\%); and cytotoxic potential: Brine shrimp cytotoxicity assay with $\mathrm{IC}_{50}(10.40 \mu \mathrm{g} / \mathrm{mL})$. BBSNiONPs restricted the bacterial and fungal pathogenic growths at 1000, 500 and $100 \mu g / m L$. Additionally, BBS-NiONPs showed stimulatory efficacy by enhancing seed germination rate and seedling growth at 31.25 and $62.5 \mu \mathrm{g} / \mathrm{mL}$. In aggregate, BBS extract has a potent antioxidant activity which makes the green biosynthesis of NiONPs easy, economical and safe. The biochemical potential of BBS-NiONPs can be useful in various biomedical and agricultural fields.

Keywords: NiONPs; green synthesis; antioxidants; antimicrobial; cytotoxicity; nano fertiliser

\section{Introduction}

Nanotechnology is revolutionising many industrial and technological fields due to the fact that it is possible with nanotech to orient material structures at extremely small scales, thereby extending the materials science toolkit. By using nanotechnology, materials can be 
made lighter, stronger, more reactive, better electrical conductors and more durable, among countless other characteristics. The properties of nanoparticles (NPs) can be altered by changing their size at the nanoscale which provides the capacity to use them in multidisciplinary fields including medicine [1]. Up until now, a variety of metals and metal oxide NPs have been synthesised such as silver, gold, platinum, magnesium, iron oxide, caesium oxide and zinc oxide [1,2]. Among these prepared NPs, nickel oxide NPs (NiONPs) have fascinated scientists from multiple fields due to their easy synthesis, small size, wide bandgap and semiconductor properties [3]. NiONPs have been reported with numerous fruitful applications in batteries, sensors, superconductors, magnetic materials, photocatalytic and catalytic analysis [4]. In green synthesis, three main sources, i.e., autotrophs (plant and algae), bacteria and fungi are used [3]. Plant-mediated green synthesis is getting significant value in biomedical fields due to its simplicity, easy availability, ecofriendly nature and nontoxicity, and it eradicates the prerequisite of reducing agents and energy from the external environment. Studies show that plants contain numerous valuable bioactive compounds like alkaloids, polyphenols, flavonoids, terpenoids, vitamins and minerals, which play a vital role as stabilising, capping and reducing agents during phytofabrication of NPs [5]. These phytochemicals also have significant antioxidant, anti-microbial, anti-inflammatory, chemo-preventative and cytotoxic potentials [6]. During green synthesis, phytochemicals with strong antioxidant potentials adsorb onto the NPs surface and make them effective antioxidant, antimicrobial, and cytotoxic NPs [7]. Therefore, the green synthesis of NPs serves as a useful and eco-friendly approach. The focus of green synthesis is being rapidly shifted toward medicinal plants due to their rich biological potential [8].

Plants belonging to the family Berberidaceae are catching interest to prepare NPs with great medical and phytonutritional properties, some recent examples are: [9-11]. The endemic shrub, B. balochistanica (Zralga), belongs to the family Berberidaceae and is found in the Quetta, Ziarat and Kalat regions of Balochistan, Pakistan [12]. The decoction of the underground part (root) is used for the treatment of various diseases like coughing, fever, wound healing, internal injury, eye disease, rheumatism and other infections of human beings and livestock [13-17]. Recently, the roots of this plant were studied and various secondary metabolites like berberisinol, berberine, palmatine, 8-oxoberberine, oleanolic acid, $\beta$-sitosterol, gallic acid, phenols, carotenoids and vitamins were isolated $[13,17]$. These secondary metabolites in the root were found with remarkable antioxidant, antibacterial and antifungal potentials $[17,18]$. The presence of bioactive compounds with antioxidative and antimicrobial properties makes this plant valuable to use for the green synthesis of NPs. However, there is little data on the chemical composition and antioxidant activity of B. balochistanica stem extract and its usage in green synthesis.

The aim of this study was to characterise the phytochemical and antioxidant activities of stem extract of B. balochistanica. After confirmation of biomolecules with significant antioxidant activities, the extract was subjected to synthesise NiONPs. The synthesised NiONPs were characterised using UV-visible, Fourier transform infrared (FTIR), energy dispersion spectroscopy (EDS), X-ray diffractometer (XRD) and scanning electron microscopy (SEM) analysis. To evaluate the biological potential of BBS-NiONPs, different in vitro biological and biofertiliser activities including antimicrobial, antioxidant, cytotoxic, inhibitory and biostimulatory activities were assessed.

\section{Results}

\subsection{Physical and Morphological Characterisation of BBS-NiONPS}

The confirmation of BBS-NiONPs was observed by various techniques and the results were validated by preceding analysis. Figure 1a represents the UV-Vis absorption spectrum of BBS-NiONPs formations using stem extract of B. balochistanica. The peak value at $305.00 \mathrm{~nm}$ specifies the absorption of metal ions. Figure $1 \mathrm{~b}$ represents the FTIR spectra with multiple peaks belonging to bio-constituents adsorbed by the synthesised NPs. The FTIR profile of $\mathrm{NiO}$ displayed vibration at $3309.75 \mathrm{~cm}^{-1}$ for $-\mathrm{OH}$ groups, at 2943.78 and $2831.77 \mathrm{~cm}^{-1}$ indicating the C-H stretching, while other multiple peaks at 1559.94, 
$1449.04,1415.19,1114.62$ and $1021.53 \mathrm{~cm}^{-1}$ were related to $\mathrm{C}=\mathrm{C}$ and $\mathrm{C}-\mathrm{O}$ stretching for aromatic ring and polyphenols. The peak at $616.36 \mathrm{~cm}^{-1}$ displayed information about $\mathrm{NiO}$ in $\mathrm{NiNO}_{3}$. The crystalline nature of BBS-NiONPs was analysed using the XRD technique. Sample NiONPs showed crystallite size $(31.44 \mathrm{~nm})$ and the peaks matched with JCPDS Card \#: ICSD ID 00-022-1189. The plane of three clear peaks and interplanar spacing ' $\mathrm{d}$ ' were (003) at $0.241 \mathrm{~nm},(012)$ at $0.2088 \mathrm{~nm}$ and (104) at $0.1477 \mathrm{~nm}$ (Figure 1c). The XRD profile displayed a rhombohedral shape of synthesised $\mathrm{NiO}$ particles. The intense and sharp peaks exposed that NiONPs were successfully shaped in the stem broth of B. balochistanica.

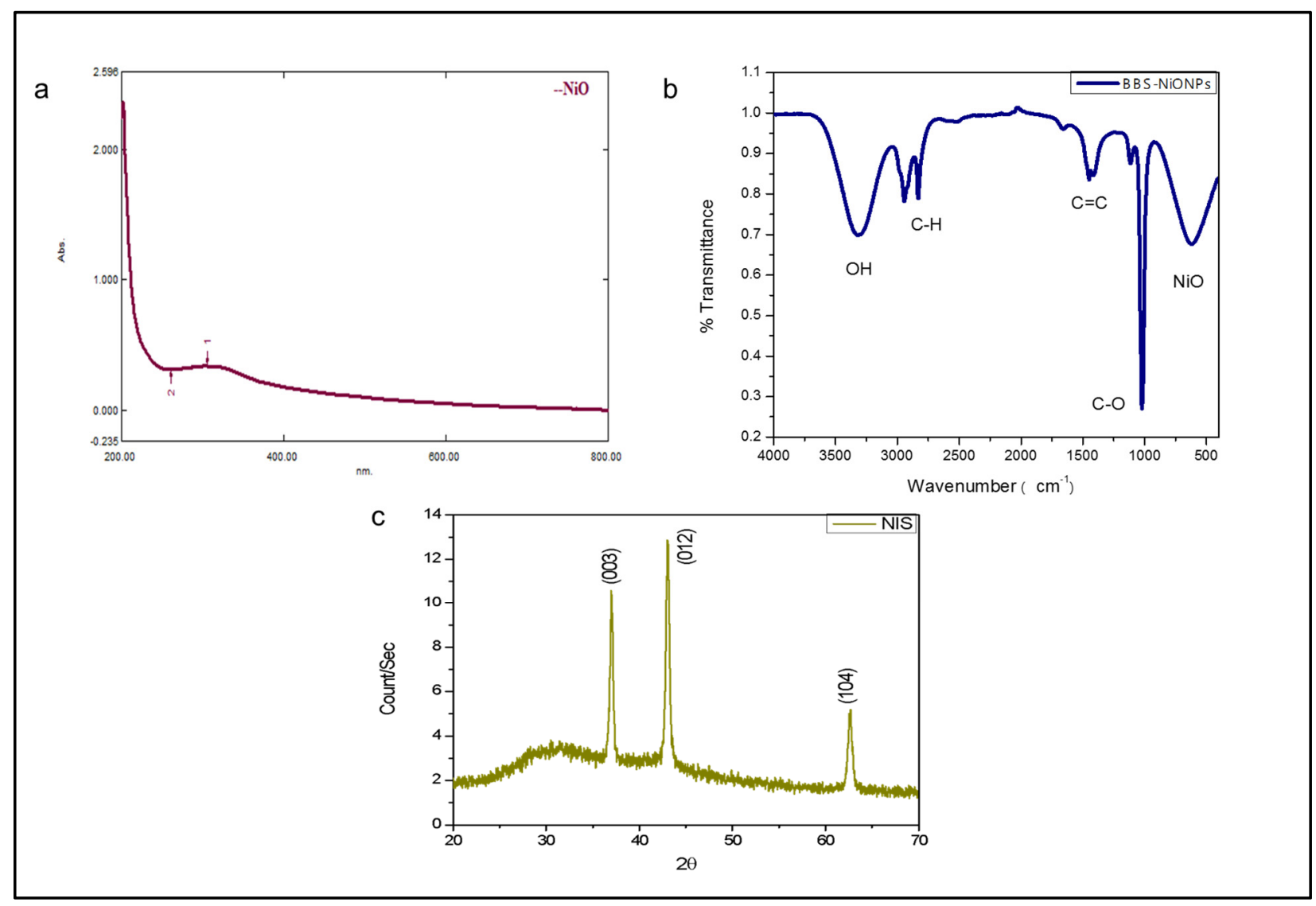

Figure 1. Spectroscopic profile of Berberis balochistanica stem (BBS)- nickel oxide nanoparticles (NiONPs). (a) UV-Vis spectrum of BBS-NiONPs. The peak value at $305.00 \mathrm{~nm}$ specifies the absorption of metal ions. (b) FTIR profile of BBSNiONPs. Peaks indicate the presence of different functional groups. (c) XRD spectrum of BBS-NiONPs.

The elemental composition of synthesised nanoparticles was confirmed by EDS analysis. Figure 2a shows strong peaks of $\mathrm{Ni}(79.19 \%)$ and $\mathrm{O}(15.56 \%)$ by weight. The presence of carbon $(4.50 \%)$ and potassium $(0.74 \%)$ in graphs was attributed to grid support. Additionally, the morphological features of greenly invented BBS-NiONPs were assessed by scanning electron microscopy (SEM). Figure $2 b$ illustrates the SEM profile of BBS-NiONPs confirming the highly agglomerated shape of the synthesised particles. The movement in suspension, for BBS-NiONPs, was studied by zeta-potential which was observed as $3.26 \mathrm{mV}$ (Figure 2c). 


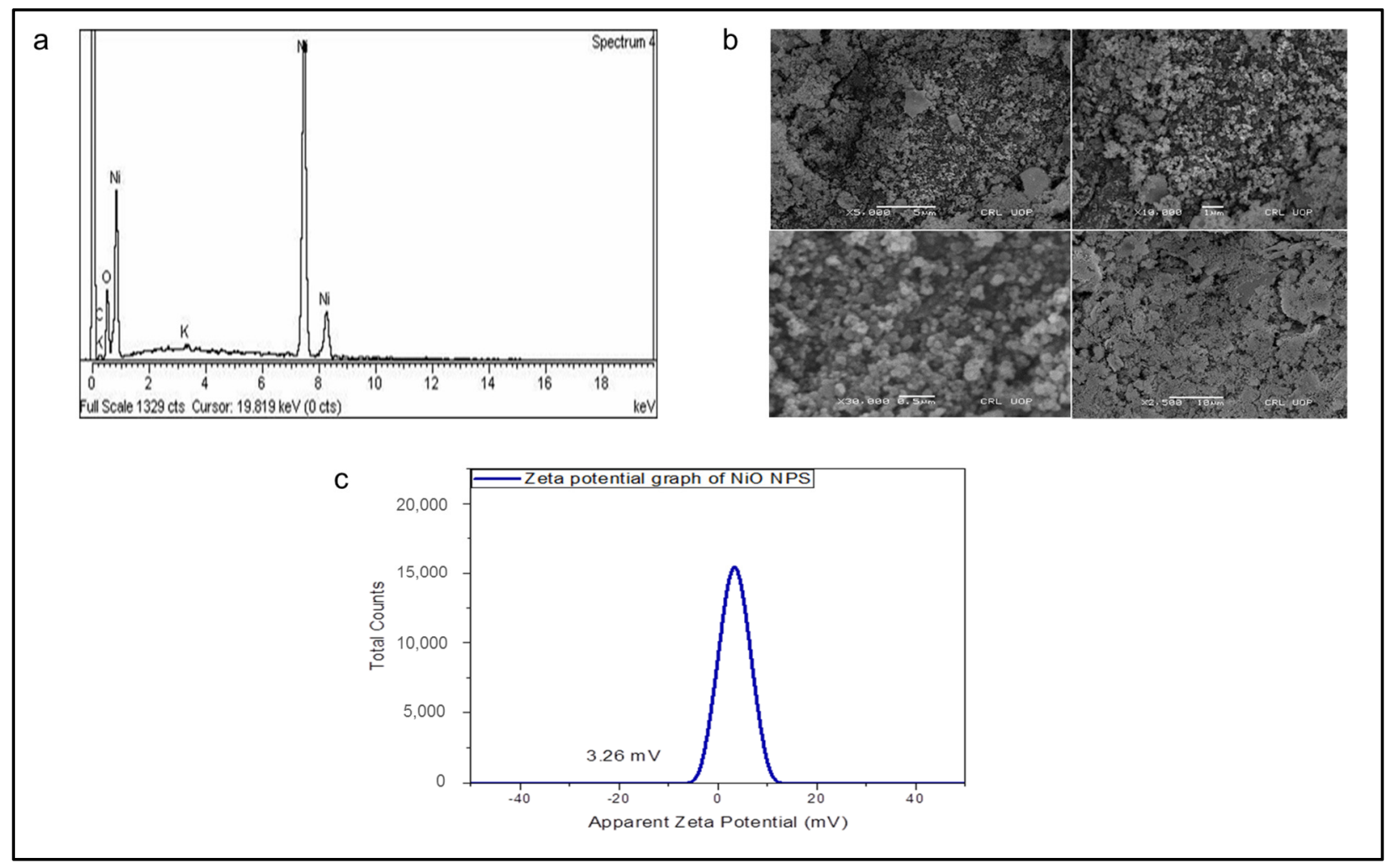

Figure 2. Spectroscopic and microscopic profile of BBS-NiONPs. (a) Energy dispersion spectroscopy (EDS) profile shows the elemental composition of BBS-NiONPs. Peaks are observed for Ni, O, C and K. (b) Micrograph of SEM shows the crystalline structure of BBS-NiONPs. (c) Zeta potential of BBS-NiONPs.

\subsection{Phytochemical and Antioxidant Analysis}

In the present study, the stem extract of B. balochistanica plant was first time analysed for total phenolic contents (TPC) and total flavonoid contents (TFC) (Figure 3a). The concentrations of TPC and TFC were $48.21 \mathrm{mg} \mathrm{GA} / \mathrm{g}$ and $141.29 \mathrm{mg} \mathrm{QE} / \mathrm{g}$. The presence of an adequate amount of berberine compound was also observed using thin-layer chromatography (TLC; Figure 3b). Additionally, the presence of biological molecules in stem extract of BB was also confirmed by FTIR spectroscopy. The functional groups were separated on the basis of peak values. The spectrum profile, peak values and functional groups were compared with the IR standard chart as shown in Figure $3 \mathrm{c}$ and Table 1. The FTIR spectrum of BBS extract showed multiples peak values indicating the presence of phenols, alcohols, alkanes, alkenes, aromatic compounds, carboxylic acid and alkyl halides. Interestingly, the absence of peaks at $2220-2260 \mathrm{~cm}^{-1}$ indicated the absence of cyanide derivatives.

Table 1. FTIR analysis of B. balochistanica plant stem extract.

\begin{tabular}{cccc}
\hline Peak Values $\left.\mathbf{( c m}^{-\mathbf{1}}\right)$ & Strength $^{\mathbf{a}}$ & Functional Groups & Interpretations \\
\hline 3306.95 & Medium & $\mathrm{OH}$ & Phenol, Alcohol \\
$2947.93-2834.58$ & Medium & $\mathrm{C}-\mathrm{H}$ & Alkane \\
1655.05 & Weak & $\mathrm{C}=\mathrm{C}$ & Alkene \\
1449.31 & Weak & $\mathrm{C}=\mathrm{C}$ & $\mathrm{C}-\mathrm{O}$ \\
1114.57 & Weak & $\mathrm{C}-\mathrm{O}$ & Aromatic compounds \\
1016.4016 & Strong & $\mathrm{C}-\mathrm{Cl}$ & carboxylic acids, alcohols \\
$587.88-522.90$ & Medium & Alkyl halides, Sulphur compounds \\
\hline
\end{tabular}

${ }^{\text {a }}$ Strength of peaks in the spectrum $\left(500-4000 \mathrm{~cm}^{-1}\right)$. 


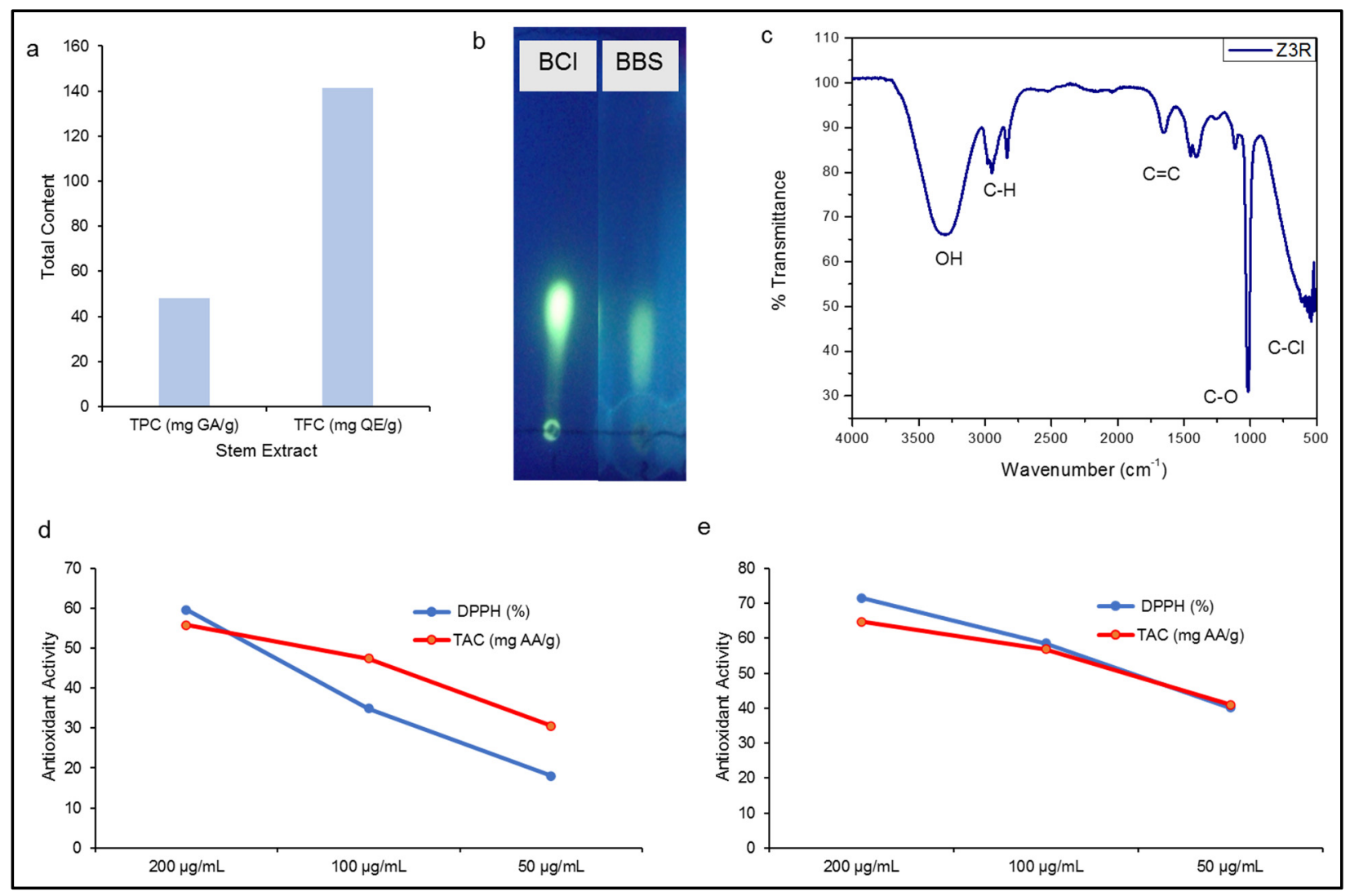

Figure 3. Phytochemical and Antioxidant analysis. (a) Total phenolic (mg GAE/g) and total flavonoid (mg QE/g) contents of stem extract. (b) Berberine in stem extract. $\mathrm{BCl}$, berberine chloride; BBS, B. balochistanica stem. (c) FTIR spectrum of stem extract. (d) Antioxidant activities (DPPH and TAC) of stem extract. (e) Antioxidant activities (DPPH and TAC) of BBS-NiONPs. Numerical data are presented as mean \pm standard deviation $(n=3)$.

Furthermore, the stem extract and green synthesised BBS-NiONPs were investigated for antioxidant potential via 2, 2-diphenyl-1-picrylhydrazyl (DPPH) and total antioxidant capacity (TAC). At $200 \mu \mathrm{g} / \mathrm{mL}$, both stem extract and BBS-NiONPs showed remarkable antioxidant activities with a percentage inhibition of 59.61 and $71.48 \%(\mathrm{DPPH})$ and 55.78 and $64.77 \%$ (TAC), respectively (Figure 3d,e). Because of the presence of these phytocompounds (TPC, TPC and berberine) and strong antioxidant activities, stem extract was selected as stabilising and capping agents for the synthesis of BBS-NiONPs in the current experiment.

\subsection{Antibacterial and Antifungal Activity of Phytofabricated NiONPs}

Figure 4a exhibits the antibacterial activity of phytofabricated BBS-NiONPs (100, 500 and $1000 \mu \mathrm{g} / \mathrm{mL}$ ) against Proteus vulgaris and Staphylococcus aureus bacterial strains. BBS-NiONPs showed a dose-dependent response against both selected bacterial strains. Moreover, $10 \mu \mathrm{g}$ Ciprofloxacin (positive control) was found more reactive than all the applied doses of BBS-NiONPs during antibacterial activities.

Antifungal analysis of BBS-NiONPs was carried out using different concentrations $(50,100,500,1000 \mu \mathrm{g} / \mathrm{mL})$ against Fusarium oxysporum, Aspergillus niger and Alternaria alternata. Dose-dependent responses against all three analysed fungus strains were observed (Figure $4 \mathrm{~b})$. F. oxysporum was found less vulnerable at high concentration $(1000 \mu \mathrm{g} / \mathrm{mL})$ while A. alternata was found highly susceptible with a percentage inhibition of $71.25 \%$ followed by $A$. niger with a percentage inhibition of $39.51 \%$ at $1000 \mu \mathrm{g} / \mathrm{mL}$. 


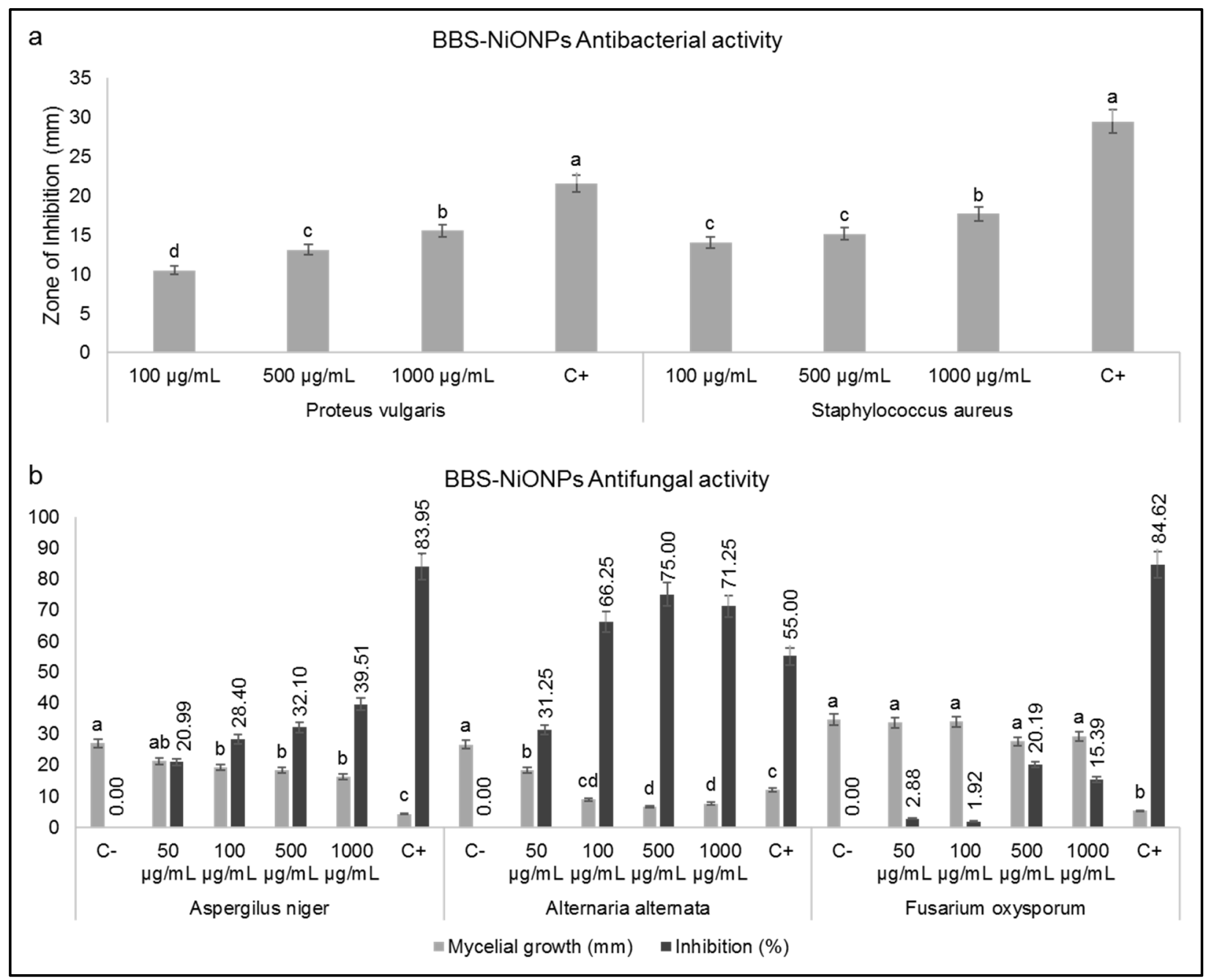

Figure 4. Antimicrobial potentials. (a) Antibacterial potential of BBS-NiONPs; C+ refers to Ciprofloxacin. (b) Antifungal potential of BBS-NiONPs. Letters indicate a significant difference $(p<0.05)$ between control and BBS-NiONPs treated samples. $\mathrm{C}+$ refers to fluconazole. Numerical data are presented as mean \pm standard deviation $(n=3)$.

\subsection{Cytotoxic Potential of Biosynthesised BBS-NiONPs}

The cytotoxic potential of biosynthesised BBS-NiONPs was examined against brine shrimp (BS) larvae. The BS larvae were exposed to BBS-NiONPs at different concentrations $(200-1 \mu \mathrm{g} / \mathrm{mL})$ for $24 \mathrm{~h}$ and a considerable dose-dependent cytotoxic response with $\mathrm{IC}_{50}$ value $(10.40 \mu \mathrm{g} / \mathrm{mL})$ was calculated (Figure 5).

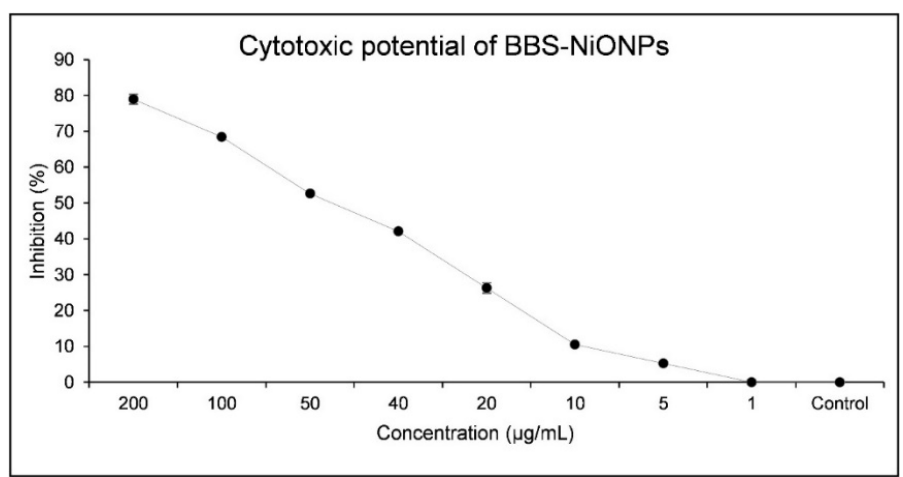

Figure 5. Brine shrimp mortality assays of BBS-NiONPs. 


\subsection{Inhibitory and Stimulatory Effect of BBS-NiONPS}

Figure 6a signifies the percentage inhibition of seed germination at the applied concentration of BBS-NiONPs $(31.25-1000 \mu \mathrm{g} / \mathrm{mL})$. The percentage inhibition was observed in a dose-dependent manner. At lower concentrations (31.25-125 $\mu \mathrm{g} / \mathrm{mL})$, the seed germination was not inhibited, while at higher concentrations, the seed germination was inhibited as 16 , 25 and $41 \%$ at 250, 500 and $1000 \mu \mathrm{g} / \mathrm{mL}$, respectively. Furthermore, the relative germination rate (RGR) of seeds during the first two days was also more fascinating as at lower doses the germination started earlier in non-treated control. The seeds treated with BBS-NiONPs (31.25 and $62.5 \mu \mathrm{g} / \mathrm{mL}$ ) showed $2-4 \%$ more germination than control (non-treated) after Day 1. After Day 2, the treated seeds ( 31.25 and $62.5 \mu \mathrm{g} / \mathrm{mL}$ ) showed 3-8\% stimulatory activity as compared to control (Figure $6 \mathrm{~b}$ ). In short, BBS-NiONPs showed stimulatory effects at lower doses and enhanced the speed of germination as compared to control (non-treated). Similarly, NiONPs also showed positive effects on the seedling growth of the radish plant. At lower concentrations ( 31.25 and $62.5 \mu \mathrm{g} / \mathrm{mL}$ ), the seedling growth was improved by 13.86 and $7.92 \%$, while at higher concentrations, the growth of the seedling declined by $33.17,37.13$ and $39.60 \%$ at $250-1000 \mu \mathrm{g} / \mathrm{mL}$, respectively (Figure $6 \mathrm{c}, \mathrm{d}$ ).

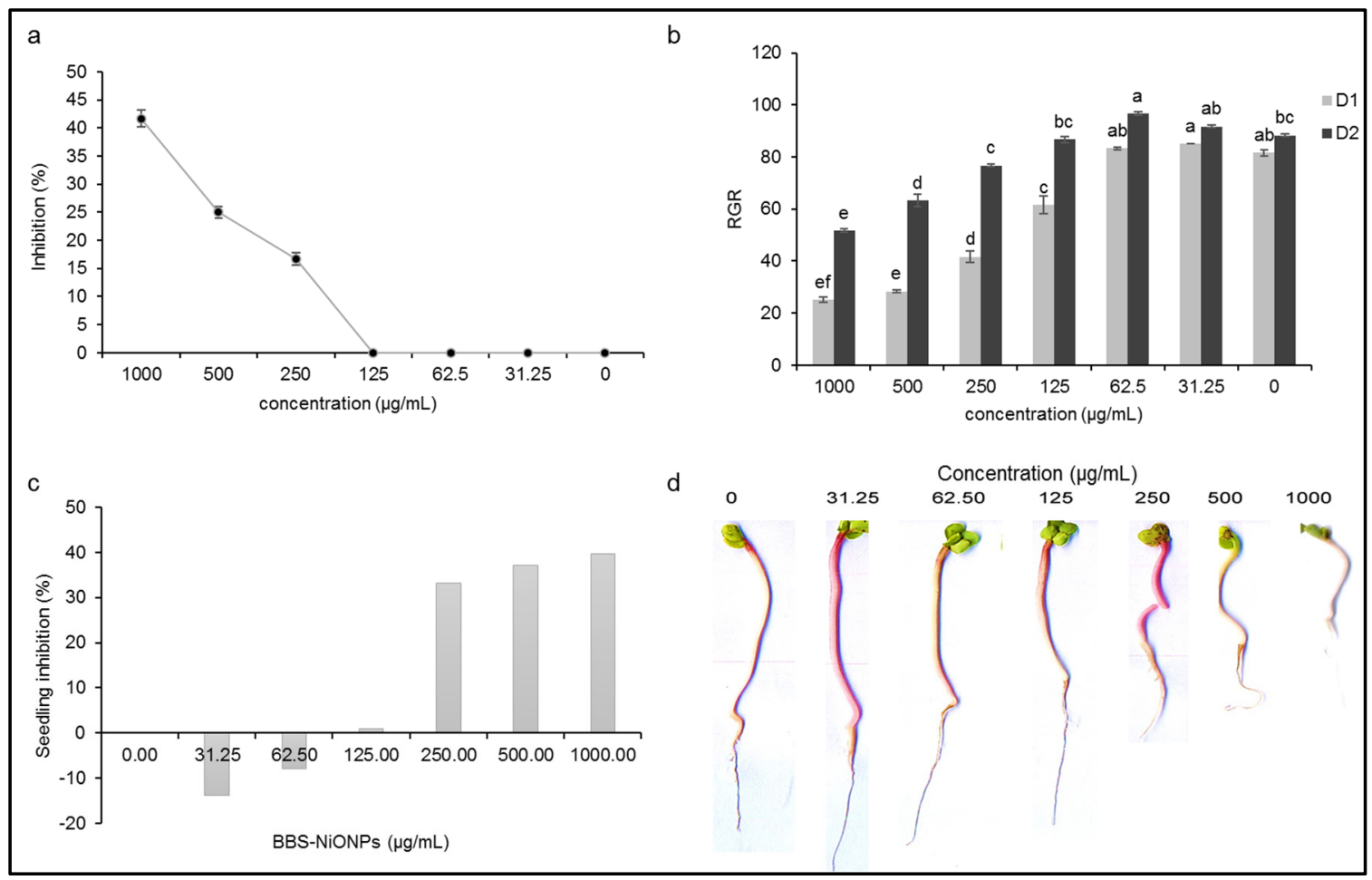

Figure 6. Effects of BBS-NiONPs on the seed germination and seedling growth of a radish plant. (a) Percentage inhibition (inhibitory effect) of seed germination against applied concentrations of BBS-NiONPs. (b) Relative germination rate (RGR) of seed (stimulatory effect) during first two days (D1 and D2). (c) Relative seedling growth rate (\%). (d) Seedling length $(\mathrm{mm})$ at applied concentrations. Letters indicate a significant difference $(p<0.05)$ between control and BBS-NiONPs treated samples. Numerical data are presented as mean \pm standard deviation $(n=3)$.

\section{Discussion}

In B. balochistanica roots, many bioactive compounds such as phenolics, pakistanamine, proaphorphin, benzylisoquinoline, alkaloid, flavanols, berberine, oleanolic acid and gallic acid have been reported $[13,14]$. In the present study, the phytochemistry of BBS was investigated and remarkable antioxidant activities were noted. FTIR of BBS indicated the presence of multiple functional groups including phenols, alcohols, alkanes, alkenes, aromatic compounds, carboxylic acid and alkyl halides. These functional groups represent 
secondary metabolites that act as a natural defense system and give medicinal properties to plants [19].

Based on the results of the present study on phytochemicals with potent antioxidant activities, the BBS extract was selected to use in the green synthesis of NiONPs. Previously, NiONPs have been synthesised by physicochemical and biological methods [20]. However, the biocompatibility and phytochemical potential of BBS-NiONPs indicate that utilising medicinal plants for green synthesis is a much better strategy to synthesise pure, safe, biocompatible and bioactive NiONPs. Although using medicinal plants for green synthesis provides rather effective NPs [11], it poses a threat to biodiversity due to the fact that many medicinal plants, such as B. balochistanica used in this study, are subject to extreme pressure due to their extensive use by local and homeopathic communities. Unlike Dangi et al. where they used B. asiatica roots for green synthesis [11], we synthesised NiONPs using B. balochistanica stem extract and found compatible, or even better, bioactivities of the synthesised NiONPs. These findings encourage using aerial parts of medicinal plants in green synthesis and other pharmacological fields-an approach that can help in the preservation of rare species.

Colour observation, spectroscopic and microscopic analysis characterised the biogenic BBS-NiONPs. The formation of BBS-NiONPs was verified by changing the colour from green to greenish-gray. The UV-Vis spectra at $305 \mathrm{~nm}$, average size $31.44 \mathrm{~nm}$, pure Ni and $\mathrm{O}$ elemental nature and rhombohedral agglomerated shape clearly validated the formation of BBS-NiONPs. Additionally, the multiple bands corroborate the presence of biomolecules on the surface of BBS-NiONPs which act as capping agents and the peak at $616.36 \mathrm{~cm}^{-1}$ is linked with Ni-O bond formation. These results show uniformity with previous reports [21-24]. The BBS-NiONPs showed agglomerated shape which represented the electrostatic interaction of the synthesised NPs with each other. It is reported that this happens due to the nano size, high surface tension, surface energy and different reducing phytochemicals in different plant extracts $[25,26]$. Due to this agglomeration, the dispersity of BBS-NiONPs became low in suspension.

The emerging resistance against antibiotics and evolving of new infectious microbial species are the main challenges for researchers. Therefore, the researchers are trying hard to develop nanomaterials with potent biological potentials against degenerative infectious diseases. The biogenic BBS-NiONPs showed astonishing DPPH radical scavenging and TAC antioxidant activities in a dose-dependent manner. As compared to BBS extract, BBS-NiONPs showed better antioxidant potential at all applied concentrations. Therefore, it is reflected that strong antioxidant activities of BBS-NiONPs are due to the interaction and adsorption of antioxidant compounds from the extract onto the surface of synthesised NPs [27]. Both bacterial isolates (gram-positive and gram-negative) were found susceptible to BBS-NiONPs. It coincides with the fact that NPs have the ability to penetrate inside the bacterial cell and obstruct metabolic activities [28]. BBS-NiONPs also showed antifungal response against tested fungal species. They inhibited mycelial growth in the following manner: A. alternata $>$ A. niger $>$ F. oxysporum. The BBS-NiONPs penetrate and enhance the permeability and generate reactive oxygen species (ROS) inside the fungal cell which retards mycelial growth [29]. The brine shrimp larvae motile movement was restricted by increasing the doses of BBS-NiONPs and ultimately mortality occurred within $24 \mathrm{~h}$. This retardation of larval movement might be due to the attachment of NPs and when the NPs penetrated inside the larval body, they reduced the metabolic activity and, as a result, mortality occurred [30]. The dose-dependent mortality rate investigations have shown that the NPs can be used as anticancer drugs in biomedical fields [22,27,31]. The remarkable antioxidant, antibacterial, antifungal and cytotoxic potentials of BBS-NiONPs might be due to their nano gage dimension, precise surface area and adherence properties.

Due to the nano size, the BBS-NiONPs displayed potent biological activities against various infectious pathogens in the current study. NPs have more attachment and penetration ability with the cell membrane of pathogens as compared to bulk materials [29]. The exact mechanism of inhibitory activity of BBS-NiONPs is not clear. However, recent 
studies show that this inhibitory potential of NPs is due to the penetration and interference of NiONPs with intracellular machinery. Briefly, the BBS-NiONPs released nickel ions which attached and penetrated inside the cell and caused leakage of the cell membrane. Inside the cell, the NiONPs generated ROS, which directly inhibited the cellular life machinery like breaking phosphate and hydrogen bonding of the DNA strand, destroying the three-dimensional structure of proteins and causing oxidative stress in the power house of the cell [32].

Recently, NPs have also been used as biofertilisers in the agricultural field for enhancing nutrients uptake, breaking seed dormancy and reducing the application of hazardous agrochemicals [33]. Therefore, green synthesis of NPs might be useful for controlled release of fertiliser, plant growth mediation and green alternative to agrochemicals. Numerous metal NPs such as $\mathrm{TiO}_{2}, \mathrm{ZnO}$ and AgNPs are used as biostimulators in the agricultural field [34]. Significant reports are available on inhibitory potentials of NiONPs, while regarding stimulatory activities, little information is available [35,36]. In the present study, BBS-NiONPs increased the seed germination (2-8\%) as compared to non-treated control, while at lower concentrations, the BBS-NiONPs showed nontoxic effects on seedling growth. In short, at a lower quantity, these green synthesised BBS-NiONPs are biocompatible and have the ability to speed up seed germination by breaking seed dormancy and can act as a growth-promoting agent. The positive response of seeds toward lower concentrations of BBS-NiONPs might be due to various factors such as infiltration of NPs, releasing ions and making a suitable environment for oxygen and water uptake, hence breaking seed dormancy and promoting seedling growth [33,37]. These findings indicate that apart from their potential in the biomedical field, green synthesised NPs could be effectively used in agricultural fields as nano fertilisers as well as stimulatory agents for plant physiology.

\section{Materials and Methods}

\subsection{Collection of Berberis balochistanica Plant}

B. balochistanica was collected during June-August 2019 from mountainous regions of Hanna Urak, Quetta, Baluchistan, Pakistan ( $\left.30^{\circ} 16^{\prime} 28.45^{\prime \prime} \mathrm{N}, 67^{\circ} 10^{\prime} 50.43^{\prime \prime} \mathrm{E}\right)$. The specimen of the collected plant was identified by comparing it with already present herbarium specimens and flora of Pakistan. The voucher specimen (RAW100268) was deposited in the National Herbarium, Islamabad, Pakistan for reference study.

\subsection{Preparation of B. balochistanica Stem Extracts}

The BBS was washed and shifted to the oven for $10 \mathrm{~h}$ at $40{ }^{\circ} \mathrm{C}$. Later, the crushed fine powder of the stem (20.66 g) was thoroughly mixed with $200 \mathrm{~mL}$ of distilled water. After stirring for $12 \mathrm{~h}$, the BBS extract was incubated in a water bath at $40{ }^{\circ} \mathrm{C}$ for $2 \mathrm{~h}$. The prepared extract was filtered three times using Whatman filter paper and centrifuged at $3000 \mathrm{rpm}$ for $30 \mathrm{~m}$ to remove the remaining aggregates. For experimental analysis, the stock solution of BBS was stored at $4{ }^{\circ} \mathrm{C}$. Primarily, we examined the phytochemical and antioxidant potential of BBS extract and then used it as a stabilising and reducing agent in the green synthesis of BBS-NiONPs.

\subsection{Phytochemical Analysis of BBS}

\subsubsection{Berberine Analysis in BBS Extract}

The presence of berberine in BBS extract was screened by TLC, $10 \mu \mathrm{L}$ of Berberine chloride $(10 \mu \mathrm{g} / \mathrm{mL})$ was used as a reference compound. A small drop of BBS extract and the reference drug were drawn using a capillary tube on a preactivated TLC plate $(8 \times 8 \mathrm{~cm})$. The TLC plate was put into the TLC tank having a mobile phase (methanol:ethyl acetate:acetic acid:water) (5:4:1:1, v/v). After separation, the TLC plate was dried and visualised by UV-Vis spectrum at $365 \mathrm{~nm}$. 


\subsubsection{Total Phenolics and Total Flavonoids Contents Analysis}

The TPC was determined in stem extract using Folin-Ciocalteu reagent [38]. In brief, $20 \mu \mathrm{L}$ of root extract was mixed with $90 \mu \mathrm{L}$ of Folin-Ciocalteu reagent, and then with $90 \mu \mathrm{L}$ of $\mathrm{NaCO}_{3}$ solution. After incubation at room temperature for $60 \mathrm{~m}$, absorbance was measured. TPC was expressed as Gallic acid equivalents (mg GAE/g) of the sample. TFC was estimated using the Aluminium Chloride Colorimetric method with some modifications. TFC in stem extract was expressed as Quercetin equivalents (mg of QE/g) of the extract.

\subsection{Green Synthesis and Physical Characterisation of BBS-NiONPS 4.4.1. BBS-NiONPs Synthesis}

For the green synthesis of BBS-NiONPs, formerly used protocols with slight changes were used [27]. To synthesise BBS-NiONPs, $50 \mathrm{~mL}$ purified BBS extract solution was steadily added to the solution of $\mathrm{NiNO}_{3}(0.3 \mathrm{M})$. The mixture was subjected to heating at $60{ }^{\circ} \mathrm{C}$ with proper stirring at $500 \mathrm{rpm}$ for $3 \mathrm{~h}$. The precipitated pellet of BBS-NiONPs was collected after centrifugation at $3000 \mathrm{rpm}$ for $25 \mathrm{~m}$ and washed with distilled water (three times). The presumptuous pellet of BBS-NiONPs was incubated at $100{ }^{\circ} \mathrm{C}$ to entirely evaporate the remaining water. Finally, the synthesised NiONPs were physically and biologically characterised using different techniques.

\subsubsection{Characterisation of BBS-NiONPs}

The bio-reduction of $\mathrm{NiNO}_{3}$ into NiONPs was confirmed by the colour changing and optical properties of this reduced solution were inveterate via absorption spectra using UV-400 UV-Vis spectrophotometer (Germany) at a scanning range of 200 to $700 \mathrm{~nm}$. FTIR at scanning range $400-4000 \mathrm{~cm}^{-1}$ was used to verify the capping and stabilising properties of various functional groups associated with the green synthesis of NPs. Additionally, the structural, elemental, vibrational and morphological nature of BBS-NiONPs were studied using XRD, Raman spectroscopy and SEM (NOVA FEISEM-450 fortified with EDX apparatus). The size of biosynthesised NiONPs was calculated by the Scherrer equation.

$$
\mathrm{D}=\mathrm{k} \lambda / \beta \cos \theta
$$

\subsection{Antioxidant Activity of BBS and BBS-NiONPS}

\subsubsection{DPPH (2,2-Diphenyl-1-picrylhydrazyl) Assay}

In this method, the in vitro free radical-scavenging potential of BBS and BBS-NiONPs at different concentrations $(50,100$ and $200 \mu \mathrm{g} / \mathrm{mL}$ ) was determined using a microplate reader [39]. Reagent solution was prepared by adding $2.4 \mathrm{mg}$ of DPPH to $25 \mathrm{~mL}$ of methanol. The procedure involved the addition of $180 \mu \mathrm{L}$ of reagent solution into $20 \mu \mathrm{L}$ of the test sample to make $200 \mu \mathrm{L}$ of the final reaction mixture. The mixture was subjected to a shaker followed by incubation for $1 \mathrm{~h}$. Ascorbic acid was used as a reference antioxidant. DPPH solution without sample was taken as control and methanol was used as a blank solution. Finally, the absorbance of the control and tested samples was measured at $517 \mathrm{~nm}$ using a microplate reader to find radical scavenging activity using the following formula below:

$$
\mathrm{DPPH} \text { scavenging effect } \%=\frac{\mathrm{AC}-\mathrm{AN}}{\mathrm{AC}} \times 100
$$

where, AC and AN refer to the absorbance of the control and NPs at $517 \mathrm{~nm}$.

\subsubsection{Total Antioxidant Capacity}

The TAC of BBS and BBS-NiONPs was evaluated by the phosphomolybdenum method [40]. NiONPs and the reagent solution $(0.6 \mathrm{~mol} / \mathrm{L}$ sulfuric acid, $28 \mathrm{mmol} / \mathrm{L}$ sodium phosphate and $4 \mathrm{mmol} / \mathrm{L}$ ammonium molybdate) were mixed and incubated at $95^{\circ} \mathrm{C}$ for $90 \mathrm{~m}$. The solution was cooled, and the absorbance of the mixture was taken at $695 \mathrm{~nm}$. TAC was calculated as $\mu \mathrm{g} / \mathrm{mg}$ equivalent of ascorbic acid ( $\mu \mathrm{g} \mathrm{AA} / \mathrm{mg}$ ). 
4.6. Anti-Microbial Analysis of BBS-NiONPs

\subsubsection{Antibacterial Screening Using Disc Diffusion Method (DDM)}

The disc diffusion method was used for antibacterial screening of green synthesised BBS-NiONPs against gram-negative (P. vulgaris) and gram-positive strains (S. aureus). Bacterial strains used in the present study were obtained from the culture bank (hospital isolates) of the Microbiology Laboratory, Faculty of Biological Science, Quaid-i-Azam University, Islamabad, Pakistan. Both strains were characterised by biochemical and cultural assessment [41]. Firstly, the Muller-Hinton agar was prepared, autoclaved and finally, the cooled media was poured into Petri plates. After solidifying, the bacterial strains were streaked out and a paper disc holding different concentrations of BBS-NiONPs $(100,500,1000 \mu \mathrm{g} / \mathrm{mL}$ ) and $10 \mu \mathrm{L}$ of Ciprofloxacin (positive control) were put on bacterial cultures. The prepared plates were incubated at $37^{\circ} \mathrm{C}$ overnight and bactericidal activity of plant-made BBS-NiONPs was observed for the zone of inhibition $(\mathrm{mm})$ around the coated discs.

\subsubsection{Antifungal Assay Using Poisoned Food Method (PFM)}

The poisoned food method (PFM) was used to study the antifungal activity of BBSNiONPs using different phytopathogenic fungal pathogens (A. niger, A. alternata and F. oxysporum). All fungal pathogens were obtained from Plant Pathology Laboratory, Department of Plant Sciences, Quaid-i-Azam University, Islamabad, Pakistan [29]. These fungal pathogens were cultured using autoclaved Sabouraud dextrose agar media (SDA; Oxoid CMO147). Different doses (100, 500 and $1000 \mu \mathrm{g} / \mathrm{mL})$ of prepared BBS-NiONPs solution were mixed with SDA media, shaken properly, and poured into Petri plates for solidification. A disc of 7 days old fungal culture $(5 \mathrm{~mm})$ was put in the middle of the media plates. The non-treated SDA media and fluconazole-treated SDA media were used as the negative and positive controls, correspondingly. After incubation for 3 days at $27^{\circ} \mathrm{C}$, the mycelial growth against various concentrations of BBS-NiONPs was measured in millimetres and the percentage of inhibition was calculated by the given formula [42].

$$
\text { Percentage Inhibition }=\mathrm{FC}-\frac{\mathrm{FN}}{\mathrm{FC}} \times 100
$$

where, FC and FN represent the average increase in fungal growth $(\mathrm{F})$ in the control and each treatment (NPs).

\subsection{Cytotoxic Assay of BBS-NiONPs by PFM}

\subsubsection{Brine Shrimp Cytotoxicity Assay (BSCA) of BBS-NiONPs}

The cytotoxic effects of BBS-NiONPs were evaluated using the brine shrimp cytotoxicity assay. For this purpose, artificial seawater was prepared by using $3.8 \mathrm{~g}$ sea salt in $1 \mathrm{~L}$ distilled water in a hatching chamber having a partition. Eggs of brine shrimp (Artemia salina) were put in a covered portion of the chamber and incubated for $48 \mathrm{~h}$ at $30{ }^{\circ} \mathrm{C}$. After hatching, 20 mature brine shrimp larvae were shifted to individual glass vials having various amounts of BBS-NiONPs $(200-1 \mu \mathrm{g} / \mathrm{mL})$ and the final volume was adjusted up to $5 \mathrm{~mL}$ by adding seawater. After $24 \mathrm{~h}$, alive brine shrimps were counted [43]. Lethality concentration ( $\mathrm{LC}_{50}$ values) and percentage mortality were calculated using GraphPad software.

\subsubsection{Phytotoxicity Assay of BBS-NiONPs}

The phytotoxic effect of green-made NPs was evaluated using the radish seed assay method (RSA) [44]. Different concentrations (31.25-1000 $\mu \mathrm{g} / \mathrm{mL})$ of BBS-NiONPs solution were introduced in each Petri plate containing sterilised filter paper (Whatman filter paper) and 15 seeds. The obtained data was measured as mean \pm standard deviation $(n=3)$ and different seed germination indices (final germination, percentage inhibition) were calculated [45]. Finally, the seedling length was measured in $\mathrm{mm}$. 


\subsection{Statistical Analysis}

The obtained data were reported in triplicates and quantified as mean \pm standard deviation. To check the inhibitory potential of BBS-NiONPs on bacterial, fungal and seedlings growth, a statistical valuation was performed by one-way analysis of variance. Multiple comparisons between means were estimated by LSD test at $95 \%$ confidence interval. All statistical analyses were performed using Statistix version 10 . The cytotoxic activity of BBS-NiONPs was estimated by calculating the lethality concentration $\left(\mathrm{IC}_{50}\right)$ values using the probit analysis (GraphPad software, San Diego, CA, USA) [46,47].

\section{Conclusions}

The present study reports the green synthesis of BBS-NiONPs from the stem extract of the Berberis balochistanica plant. The presence of valuable phytochemicals with bioactive functional groups and potent antioxidants in stem extract helped in stabilising, capping and reducing nickel salt into BBS-NiONPs. The crystalline rhombohedral shape and fine size $(31.44 \mathrm{~nm})$ of BBS-NiONPs were confirmed by SEM and XRD techniques. Remarkable biological applications of BBS-NiONPs, like antioxidant, antimicrobial and cytotoxic potentials, were observed. BBS-NiONPs were also found as biostimulators in boosting up the germination frequency and seedling growth at suitable quantities. This indicates that they could be used as a substitute for synthetic chemicals in biomedical and agricultural fields. Moreover, they are also suitable for plants with high dormancy and slow seed germination. However, a further widespread investigation is recommended before introducing BBS-NiONPs into clinical and agricultural trials.

Author Contributions: Conceptualization, S.U.; Data curation, S.U. and S.L.; Formal analysis, S.A., M.S.S. and A.R.; Funding acquisition, Y.W. and U.M.Q.; Investigation, S.U., L.B.S., J.I., S.L., M.A. and A.R.; Methodology, S.U., J.I., B.A.A., M.S.S. and M.A.; Project administration, L.B.S., Y.W. and U.M.Q.; Resources, L.B.S., Y.W. and U.M.Q.; Software, S.U. and S.A.; Supervision, U.M.Q.; Validation, L.B.S., S.L., B.A.A. and A.R.; Visualization, S.U. and L.B.S.; Writing-original draft, S.U. and L.B.S.; Writing-review \& editing, L.B.S., J.I., A.B., Y.W. and U.M.Q. All authors have read and agreed to the published version of the manuscript.

Funding: This research received no external funding.

Institutional Review Board Statement: Not applicable.

Informed Consent Statement: Not applicable.

Data Availability Statement: All data used for the present study has been provided within the manuscript. For further assistance, if required, please contact the corresponding author(s).

Acknowledgments: We thank Amir Sultan (National Herbarium, Islamabad, Pakistan) for helping in the identification of plant specimens.

Conflicts of Interest: The authors declare no conflicts.

\section{References}

1. Chandra, H.; Patel, D.; Kumari, P.; Jangwan, J.; Yadav, S. Phyto-mediated synthesis of zinc oxide nanoparticles of Berberis aristata: Characterization, antioxidant activity and antibacterial activity with special reference to urinary tract pathogens. Mater. Sci. Eng. 2019, 102, 212-220. [CrossRef]

2. Iqbal, J.; Abbasi, B.A.; Ahmad, R.; Mahmood, T.; Ali, B.; Khalil, A.T.; Kanwal, S.; Shah, S.A.; Alam, M.M.; Badshah, H. Nanomedicines for developing cancer nanotherapeutics: From benchtop to bedside and beyond. Appl. Microbiol. Biotechnol. 2018, 102, 9449-9470. [CrossRef] [PubMed]

3. Iqbal, J.; Abbasi, B.A.; Ahmad, R.; Mahmoodi, M.; Munir, A.; Zahra, S.A.; Shahbaz, A.; Shaukat, M.; Kanwal, S.; Uddin, S. Phytogenic Synthesis of Nickel Oxide Nanoparticles (NiO) Using Fresh Leaves Extract of Rhamnus triquetra (Wall.) and Investigation of Its Multiple In Vitro Biological Potentials. Biomedicines 2020, 8, 117. [CrossRef] [PubMed]

4. Mayedwa, N.; Mongwaketsi, N.; Khamlich, S.; Kaviyarasu, K.; Matinise, N.; Maaza, M. Green synthesis of nickel oxide, palladium and palladium oxide synthesized via Aspalathus linearis natural extracts: Physical properties \& mechanism of formation. Appl. Surf. Sci. 2018, 446, 266-272. 
5. Abbasi, B.A.; Iqbal, J.; Kiran, F.; Ahmad, R.; Kanwal, S.; Munir, A.; Uddin, S.; Nasir, J.A.; Chalgham, W.; Mahmood, T. Green formulation and chemical characterizations of Rhamnella gilgitica aqueous leaves extract conjugated NiONPs and their multiple therapeutic properties. J. Mol. Struct. 2020, 1218, 128490. [CrossRef]

6. Batool, F.; Saadullah, M.; Asif, M.; Uzair, M.; Choudhary, B.A.; Afzal, S.; Tareen, R.B. Phytochemical and Biological Screening of Root Extracts of Berberis baluchistanica. BioCell 2019. Available online: https:/ / www.semanticscholar.org/paper/Phytochemicaland-biological-screening-of-root-of-Batool-Saadullah/8d55327f51b0ea12221fb3db8dacefcbd9b41b06 (accessed on 13 January 2021).

7. Phull, A.-R.; Abbas, Q.; Ali, A.; Raza, H.; Zia, M.; Haq, I.-U. Antioxidant, cytotoxic and antimicrobial activities of green synthesized silver nanoparticles from crude extract of Bergenia ciliata. Future J. Pharm. Sci. 2016, 2, 31-36. [CrossRef]

8. Chandra, H.; Kumari, P.; Bontempi, E.; Yadav, S. Medicinal plants: Treasure trove for green synthesis of metallic nanoparticles and their biomedical applications. Biocatal. Agric. Biotechnol. 2020, 24, 101518. [CrossRef]

9. Behravan, M.; Panahi, A.H.; Naghizadeh, A.; Ziaee, M.; Mahdavi, R.; Mirzapour, A. Facile green synthesis of silver nanoparticles using Berberis vulgaris leaf and root aqueous extract and its antibacterial activity. Int. J. Biol. Macromol. 2019, 124, 148-154. [CrossRef] [PubMed]

10. Nasrollahzadeh, M.; Maham, M.; Rostami-Vartooni, A.; Bagherzadeh, M.; Sajadi, S.M. Barberry fruit extract assisted in situ green synthesis of $\mathrm{Cu}$ nanoparticles supported on a reduced graphene oxide-Fe3O4 nanocomposite as a magnetically separable and reusable catalyst for the O-arylation of phenols with aryl halides under ligand-free conditions. RCS Adv. 2015, 5, 64769-64780.

11. Dangi, S.; Gupta, A.; Gupta, D.K.; Singh, S.; Parajuli, N. Green synthesis of silver nanoparticles using aqueous root extract of Berberis asiatica and evaluation of their antibacterial activity. Chem. Data Collect. 2020, 28, 100411. [CrossRef]

12. Khan, T.; Khan, I.A.; Rehman, A. A review on Berberis species reported from Gilgit-Baltistan and Central Karakoram National Park, Pakistan. J. Med. Plants 2014, 2, 16-20.

13. Pervez, S.; Saeed, M.; Ali, M.S.; Fatima, I.; Khan, H.; Ullah, I. Antimicrobial and Antioxidant Potential of Berberisinol, a New Flavone from Berberis baluchistanica. Chem. Nat. Compd. 2019, 55, 247-251. [CrossRef]

14. Kakar, S.A.; Tareen, R.B.; Kakar, M.A.; Jabeen, H.; Kakar, S.; Al-Kahraman, Y.; Shafee, M. Screening of antibacterial activity of four medicinal plants of Balochistan-Pakistan. Pak. J. Bot. 2012, 44, 245-250.

15. Khan, I.; Najeebullah, S.; Ali, M.; Shinwari, Z.K. Phytopharmacological and ethnomedicinal uses of the Genus Berberis (Berberidaceae): A review. Trop. J. Pharm. Res. 2016, 15, 2047-2057. [CrossRef]

16. Bibi, T.; Ahmad, M.; Tareen, R.B.; Tareen, N.M.; Jabeen, R.; Rehman, S.-U.; Sultana, S.; Zafar, M.; Yaseen, G. Ethnobotany of medicinal plants in district Mastung of Balochistan province-Pakistan. J. Ethnopharmcol. 2014, 157, 79-89. [CrossRef] [PubMed]

17. Baloch, N.; Nabi, S.; Yasser, M.; Kahraman, A. In vitro antileishmanial, cytotoxic, anti-oxidant activities and phytochemical analysis of Berberis baluchistanica roots extracts and its fractions. Phytopharmacology 2013, 4, 282-287.

18. Dastagir, G.; Pervez, N. Elemental composition of Alstonia scholaris Linn. Pak. J. Plant Sci. 2004, 10, 47-50.

19. Dhivya, K. Screening of phytoconstituents, UV-VIS Spectrum and FTIR analysis of Micrococca mercurialis (L.) Benth. Int. J. Herb. Med. 2017, 5, 40-44.

20. Ádám, A.A.; Szabados, M.; Varga, G.; Papp, Á.; Musza, K.; Kónya, Z.; Kukovecz, Á.; Sipos, P.; Pálinkó, I. Ultrasound-assisted hydrazine reduction method for the preparation of nickel nanoparticles, physicochemical characterization and catalytic application in Suzuki-Miyaura cross-coupling reaction. Nanomaterials 2020, 10, 632. [CrossRef]

21. Lingaraju, K.; Naika, H.R.; Nagabhushana, H.; Jayanna, K.; Devaraja, S.; Nagaraju, G. Biosynthesis of Nickel oxide Nanoparticles from Euphorbia heterophylla (L.) and their biological application. Arab. J. Chem. 2020, 13, 4712-4719. [CrossRef]

22. Khalil, A.T.; Ovais, M.; Ullah, I.; Ali, M.; Shinwari, Z.K.; Maaza, M. Physical properties, biological applications and biocompatibility studies on biosynthesized single phase cobalt oxide (Co3O4) nanoparticles via Sageretia thea (Osbeck.). Arab. J. Chem. 2020, 13, 606-619. [CrossRef]

23. Karpagavinayagam, P.; Prasanna, A.E.P.; Vedhi, C. Eco-friendly synthesis of nickel oxide nanoparticles using Avicennia Marina leaf extract: Morphological characterization and electrochemical application. Mater. Today 2020. [CrossRef]

24. Samrot, A.V.; Senthilkumar, P.; Rashmitha, S.; Veera, P.; Sahithya, C.S. Azadirachta indica influenced biosynthesis of superparamagnetic iron-oxide nanoparticles and their applications in tannery water treatment and X-ray imaging. Nanostruct. Chem. 2018, 8, 343-351. [CrossRef]

25. Landi, B.J.; Ruf, H.J.; Evans, C.M.; Cress, C.D.; Raffaelle, R.P. Purity assessment of single-wall carbon nanotubes, using optical absorption spectroscopy. J. Phys. Chem. 2005, 109, 9952-9965. [CrossRef] [PubMed]

26. Fardood, S.T.; Ramazani, A.; Moradi, S. A novel green synthesis of nickel oxide nanoparticles using Arabic gum. Chem. J. Mold. 2017, 12, 115-118. [CrossRef]

27. Iqbal, J.; Abbasi, B.A.; Mahmood, T.; Hameed, S.; Munir, A.; Kanwal, S. Green synthesis and characterizations of Nickel oxide nanoparticles using leaf extract of Rhamnus virgata and their potential biological applications. Appl. Organomet. Chem. 2019, 33, e4950. [CrossRef]

28. Khalandi, B.; Asadi, N.; Milani, M.; Davaran, S.; Abadi, A.J.N.; Abasi, E.; Akbarzadeh, A. A review on potential role of silver nanoparticles and possible mechanisms of their actions on bacteria. Drug Res. 2017, 11, 70-76. [CrossRef] [PubMed]

29. Ali, M.; Haroon, U.; Khizar, M.; Chaudhary, H.J.; Hussain Munis, M.F. Scanning electron microscopy of bio-fabricated Fe2O3 nanoparticles and their application to control brown rot of citrus. Mic. Res. Tech. 2021, 84, 101-110. [CrossRef] [PubMed] 
30. Ates, M.; Daniels, J.; Arslan, Z.; Farah, I.O.; Rivera, H.F. Comparative evaluation of impact of Zn and ZnO nanoparticles on brine shrimp (Artemia salina) larvae: Effects of particle size and solubility on toxicity. Environ. Sci. Proc. Imp. 2013, 15, 225-233. [CrossRef]

31. Abbasi, B.A.; Iqbal, J.; Mahmood, T.; Ahmad, R.; Kanwal, S.; Afridi, S. Plant-mediated synthesis of nickel oxide nanoparticles (NiO) via Geranium wallichianum: Characterization and different biological applications. Mater. Res. Exp. 2019, 6, 0850a0857. [CrossRef]

32. Srihasam, S.; Thyagarajan, K.; Korivi, M.; Lebaka, V.R.; Mallem, S.P.R. Phytogenic generation of NiO nanoparticles using Stevia leaf extract and evaluation of their in-vitro antioxidant and antimicrobial properties. Biomolecules 2020, 10, 89. [CrossRef] [PubMed]

33. Younes, N.; Hassan, H.S.; Elkady, M.F.; Hamed, A.; Dawood, M.F. Impact of synthesized metal oxide nanomaterials on seedlings production of three Solanaceae crops. Heliyon 2020, 6, e03188. [CrossRef] [PubMed]

34. Prażak, R.; Święciło, A.; Krzepiłko, A.; Michałek, S.; Arczewska, M. Impact of Ag Nanoparticles on Seed Germination and Seedling Growth of Green Beans in Normal and Chill Temperatures. Agriculture 2020, 10, 312. [CrossRef]

35. Chaudhary, S.; Kaur, Y.; Jayee, B.; Chaudhary, G.R.; Umar, A. NiO nanodisks: Highly efficient visible-light driven photocatalyst, potential scaffold for seed germination of Vigna Radiata and antibacterial properties. J. Clean. Prod. 2018, 190, 563-576. [CrossRef]

36. Faisal, M.; Saquib, Q.; Alatar, A.A.; Al-Khedhairy, A.A.; Hegazy, A.K.; Musarrat, J. Phytotoxic hazards of NiO-nanoparticles in tomato: A study on mechanism of cell death. J. Hazard. Mater. 2013, 250, 318-332. [CrossRef] [PubMed]

37. Dawood, M.F.; Abeed, A.H.; Aldaby, E.E. Titanium dioxide nanoparticles model growth kinetic traits of some wheat cultivars under different water regimes. Plant Physiol. Rep. 2019, 24, 129-140. [CrossRef]

38. Chlopicka, J.; Pasko, P.; Gorinstein, S.; Jedryas, A.; Zagrodzki, P. Total phenolic and total flavonoid content, antioxidant activity and sensory evaluation of pseudocereal breads. Food Sci. Technol. 2012, 46, 548-555. [CrossRef]

39. Ma, X.; Wu, H.; Liu, L.; Yao, Q.; Wang, S.; Zhan, R.; Xing, S.; Zhou, Y. Polyphenolic compounds and antioxidant properties in mango fruits. Sci. Hort. 2011, 129, 102-107. [CrossRef]

40. Prieto, P.; Pineda, M.; Aguilar, M. Spectrophotometric quantitation of antioxidant capacity through the formation of a phosphomolybdenum complex: Specific application to the determination of vitamin E. Anal. Biochem. 1999, 269, 337-341. [CrossRef] [PubMed]

41. Argueta-Figueroa, L.; Morales-Luckie, R.A.; Scougall-Vilchis, R.J.; Olea-Mejía, O.F. Synthesis, characterization and antibacterial activity of copper, nickel and bimetallic $\mathrm{Cu}-\mathrm{Ni}$ nanoparticles for potential use in dental materials. J. Prog. Nat. Sci. Mater. Int. 2014, 24, 321-328. [CrossRef]

42. Singh, J.; Tripathi, N. Inhibition of storage fungi of blackgram (Vigna mungo L.) by some essential oils. Flavour Fragr. J. 1999, 14, 1-4. [CrossRef]

43. Meyer, B.; Ferrigni, N.; Putnam, J.; Jacobsen, L.; Nichols, D.j.; McLaughlin, J.L. Brine shrimp: A convenient general bioassay for active plant constituents. Planta Med. 1982, 45, 31-34. [CrossRef] [PubMed]

44. Turker, A.U.; Camper, N. Biological activity of common mullein, a medicinal plant. J. Ethnopharmacol. 2002, 82, 117-125. [CrossRef]

45. Pérez-García, F.; González-Benito, M. Seed germination of five Helianthemum species: Effect of temperature and presowing treatments. J. Arid Environ. 2006, 65, 688-693. [CrossRef]

46. Waghulde, S.; Kale, M.K.; Patil, V. Brine Shrimp Lethality Assay of the Aqueous and Ethanolic Extracts of the Selected Species of Medicinal Plants. In Proceedings of the 23rd International Electronic Conference on Synthetic Organic Chemistry, online, 5 November-15 December 2019; p. 47.

47. Hayes, A.W.; Kruger, C.L. Hayes' Principles and Methods of Toxicology; CRC Press: Boca Raton, FL, USA, 2014. 